\section{Where has the air gone?}

Pneumorrhachis (air within the spinal canal) is rare and usually associated with other air leaks. Diagnosis is usually by high resolution CT scan.

A 9-year-old boy had a 10-day history of persistent cough despite treatment with inhaled bronchodilators, systemic steroids and antibiotics before coming to hospital. Physical examination demonstrated good general condition, cervical subcutaneous emphysema and reduced breath sounds with widespread crackles and wheeze. Chest x-ray demonstrated pneumomediastinum and overinflation of the left upper lung field. CT showed pneumopericardium, pneumothorax and pneumorrhachis (figure 1). The patient recovered uneventfully and no underlying cause was found.

Pneumorrhachis is rare, particularly in children: it can be classified into internal, intradural (intraspinal air within the subdural or subarachnoid space) and external, extradural (intraspinal, epidural air). ${ }^{1} 2$

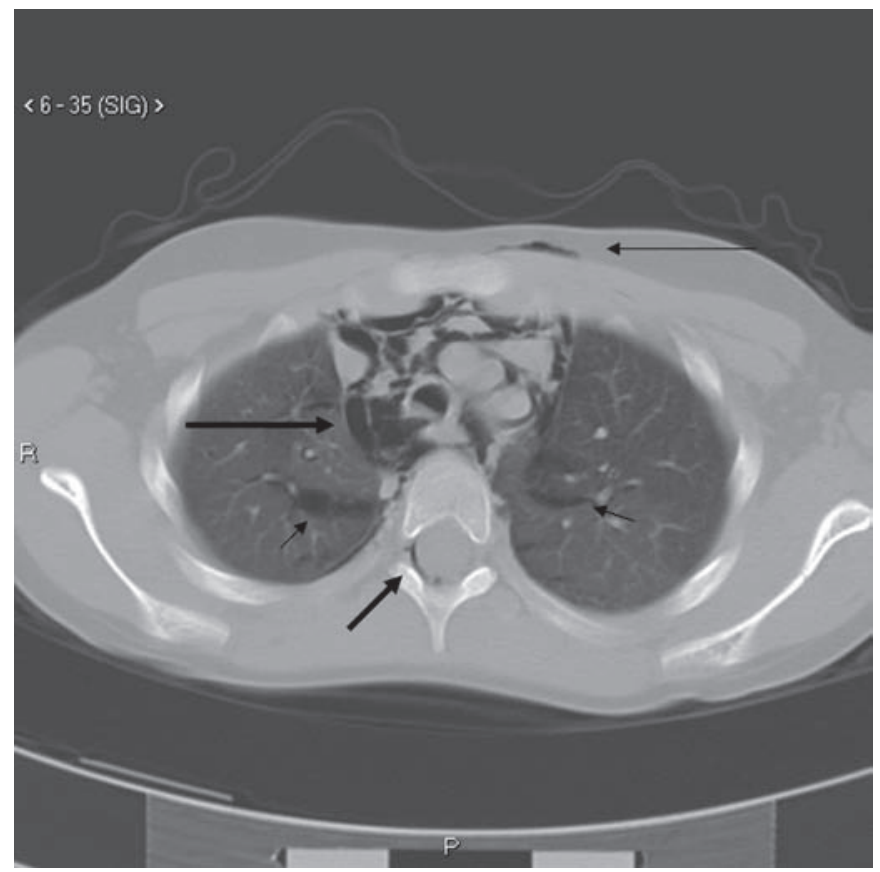

Figure 1 Axial CT image of lung apices showing free air in the right and posterior aspects of the thoracic spinal canal (short broad arrow), massive pneumomediastinum (long broad arrow), interstitial emphysema in the pulmonary apices (short slim arrows), and subcutaneous emphysema between anterior thoracic subcutaneous fat and right pectoralis major muscle (long slim arrow).
It is usually asymptomatic and can be due to trauma, extension of pneumothorax or pneumomediastinum, iatrogenic causes (lumbar puncture or epidural anaesthesia) and malignancy and its treatment. ${ }^{3-5}$ Subarachnoid pneumorrhachis can occur after trauma or extensive surgical exposure of the spinal nerve roots; it is often associated with a fatal outcome. ${ }^{3}$

In summary, we report the case of a child who developed pneumorrhachis after coughing, presumably secondary to a community acquired viral infection. Imaging showed epidural pneumorrhachis with air throughout the spinal canal.

\section{LEARNING POINTS}

- Pneumorrhachis is rare, especially in children.

- It is usually an asymptomatic epiphenomenon but can also cause neurological symptoms.

- It is seen in association with other manifestations of air leak.

- The intra-spinal air usually reabsorbs spontaneously

\section{Teresa Salerno, ${ }^{1}$ Renato Cutrera, ${ }^{1}$ Francesco Paolo Rossi, ${ }^{1}$ Laura Menchini, ${ }^{2}$ Andrew Bush ${ }^{3}$}

${ }^{1}$ Department of Pediatrics, Bronchopneumology Unit, Children's Hospital and Research Institute Bambino Gesù, Rome, Italy

${ }^{2}$ Department of Radiology, Children's Hospital and Research Institute Bambino Gesù, Rome, Italy

${ }^{3}$ Department of Pediatric Respirology, Royal Brompton Hospital, London, UK

Correspondence to Teresa Salerno, Department ofPediatrics, Bronchopneumology Unit, Children's Hospital and Research Institute Bambino Gesù, Piazza S. Onofrio 4, 00135, Rome, Italy; teresa.salerno@opbg.net

\section{Competing interests None.}

Provenance and peer review Not commissioned; externally peer reviewed.

Accepted 15 August 2010

Published Online First 30 September 2010

Arch Dis Child 2011;96:43.

doi:10.1136/adc.2010.196857

\section{REFERENCES}

1. Gibikote $\mathbf{S}$, Wray A, Fink AM. Pneumorrhachis secondary to traumatic pneumomediastinum in a child. Pediatr Radio/ 2006;36:711-13.

2. Cayli SR, Koçak A, Kutlu R, et al. Spinal pneumorrhachis. Br J Neurosurg 2003;17:72-4

3. Ristagno RL, Hiratzka LF, Rost RC, Jr, . An unusual case of pneumorrhachis following resection of lung carcinoma. Chest 2002;121:1712-14.

4. Drevelengas A, Kalaitzoglou I, Petridis A. Pneumorrhachis associated with spontaneous pneumomediastinum. Eur J Radiol 1994;18:122-3.

5. Tsuji J, Takazura E, Terada Y, et al. CT demonstration of spinal epidural emphysema complicating bronchial asthma and violent coughing. J Comput Assist Tomogr 1989;13:38-9. 